UCRL-ID-115969

\title{
Dispersion Relations \\ for a Plasma-Filled Helix-Loaded-Waveguide
}

M.A. Makowski, E.B. Hooper, and B.W. Stallard

January 1994

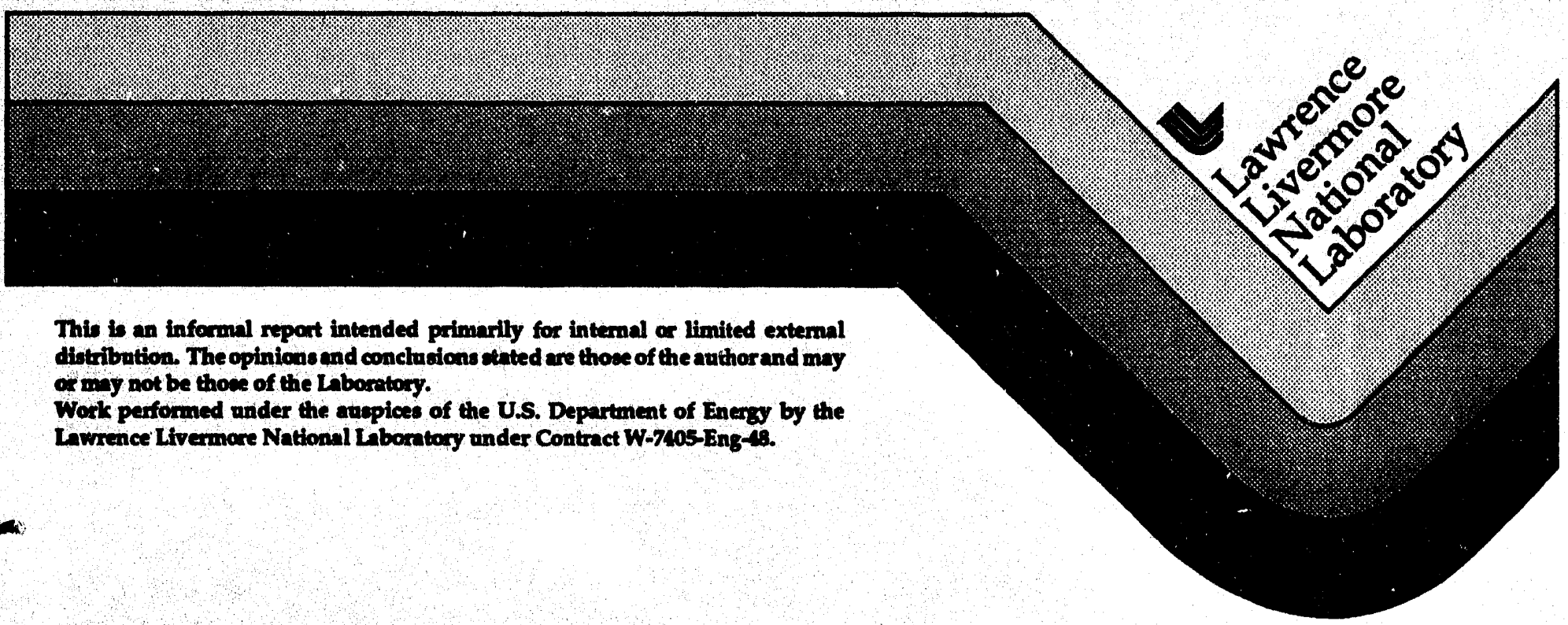

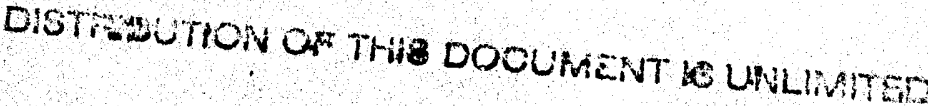




\section{Disclinmer}

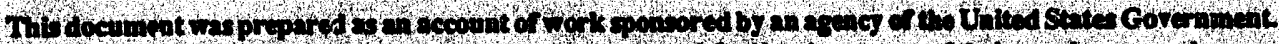

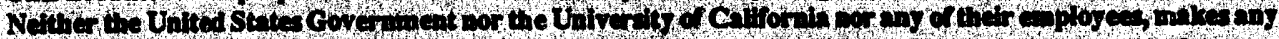

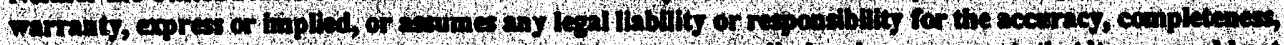
or ur

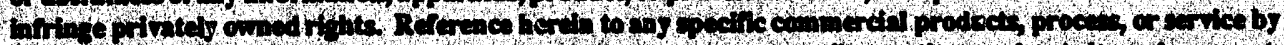

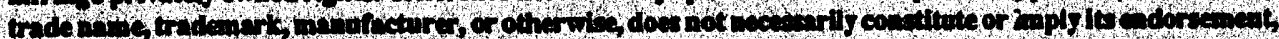

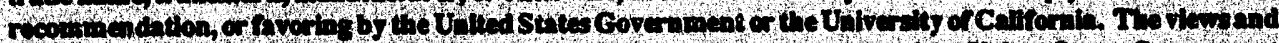

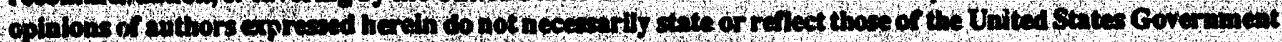

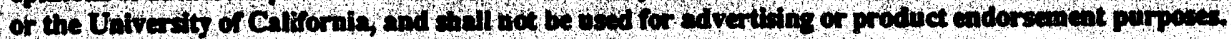

This roout has beea reprodiced directis from the bed avalibblecopy.

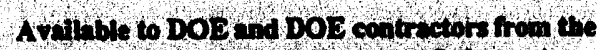
Once or Solentinc and Techilcal In formation

P.0. Ber 62, Oak Bute, TW 37031

Prices avallable from (615) 576 -8401, FIS 626-8401

Available to the publie trom the

National Techaicl Informadion Sarvice

US. Department of Commeree

5285 Port Rojal Rd,

Springrield, VA 22161 


\title{
Dispersion Relations for a Plasma-Filled Helix-Loaded-Waveguide
}

\author{
M.A. Makowski, E.B. Hooper, B.W. Stallard
}

The propagation of waves on bounded, magnetized plasma columns arises in connection with a variety of applications. To this end dispersion relations are developed for a variety of multi-region circularly symmetric configurations. These include, a sheath helix in free space, a plasma column in free space, a plasma filled conducting tube, a plasma filled sheath-helix in free space, a sheath helix within a conducting cylinder, a plasma filled sheath-helix within a conducting cylinder, and a plasma column within a sheath-helix contained within a conducting cylinder. The latter configuration is of the most interest for whistler wave excitation for plasma thruster applications, since it includes the effect of a vacuum region separating the plasma column from the helical excitation structure.

\section{Introduction}

High-frequency electromagnetic waves on magnetized plasma columns are of interest for a variety of applications. In the frequency range in which these waves interact primarily with electrons, they are used to generate and heat plasmas and, in some cases, to take advantage of distortions in the electron velocity distribution function.

Inductive excitation of waves can be accomplished using antennas outside the plasma column. Helicon waves excited this way have been used to generate cool, dense plasmas. ${ }^{1-4}$ These plasmas are of particular interest for plasma processing, ${ }^{5,6}$ generation of plasmas for lasers, ${ }^{7}$ and as a medium for wave acceleration of ions to high energy. ${ }^{8}$

Whistler waves propagate on the same branch of the plasma dispersion relation as helicons but at a sufficiently high frequency that their characteristics are affected by the cyclotron resonance. Excitation of these waves has usually been done by launching them along the fieldlines from a microwave horn or similar launcher. ${ }^{9-13}$ The present authors are conducting an experiment, ${ }^{14,15}$ to excite whistler waves on a column by inductive excitation from the edge; energy is absorbed when the waves propagate down the magnetic field to the resonance. The resulting expansion of the anisotropic, nonthermal plasma in a magnetic nozzle can impart momentum to a structure through the magnetic field, thus 
generating thrust. The gual is a high exhaust-velocity rocket of interest for near-earth and interplanetary travel.

As part of this effort, we need to understand the propagation and excitation of these waves on a uniform plasma column. In both the model and the experiment the plasma is surrounded by an annular region with a constant dielectric constant inside a cylindrical metallic waveguide. A helical antenna used to couple slow waves to the plasma modes is modeled in the analysis. The plasma is represented in the cold, high frequency approximation and ion motion is neglected. The resulting formalism holds for electron-wave excitation at all high frequencies.

We focus primarily on deriving the dispersion relation for waves in the full multi-region cylindrical system. The dispersion relations developed below thus provide an understanding of the bounded plasma/waveguide normal modes in more complicated geometries than previously studied so that efficient coupling schemes can be developed. The results are compared with previous calculations in the appropriate limits, providing a connection with existing work. The details of the coupling in parameter regimes of interest to the experiment, and of other features characteristic to applications, will be based on these results but presented in a separate publication.

\section{Field Equations for Regions of Uniform and Anisotropic Dielectrics}

\section{A. Plasma Model}

In the dispersion relations to be developed below there will be one or more radially separated regions. As shown in Fig. 1, each region is filled with either a uniform isotropic dielectric or a uniformly magnetized plasma described by an anisotropic dielectric tensor. Boundary conditions at the interfaces couple the waves in adjacent regions.

For the purpose of this paper we neglect the ion motion. However, we use this model only when evaluating the dispersion relations and resulting field profiles. Other plasma models consistent with the form of the dielectric tensor adopted below can also be substituted. The cold plasma dielectric tensor valid at electron cyclotron frequencies is

$$
\epsilon=\left(\begin{array}{ccc}
\epsilon_{\perp} & \epsilon_{\mathbf{x}} & 0 \\
\epsilon_{\mathrm{x}} & \epsilon_{\perp} & 0 \\
0 & 0 & \epsilon_{z}
\end{array}\right)
$$

is assumed in which

$$
\epsilon_{z}=1-\frac{\omega_{p}^{2}}{\omega^{2}} \quad \epsilon_{\perp}=1-\frac{\omega_{p}^{2}}{\omega^{2}-\omega_{c}^{2}}
$$




$$
\begin{gathered}
\epsilon_{\mathrm{x}}=-i \frac{\omega_{c}}{\omega} \frac{\omega_{p}^{2}}{\omega^{2}-\omega_{c}^{2}} \\
\epsilon_{R}=1-\frac{\omega_{p}^{2}}{\omega\left(\omega-\omega_{c}\right)} \quad \epsilon_{L}=1-\frac{\omega_{p}^{2}}{\omega\left(\omega+\omega_{c}\right)} \\
\epsilon_{R} \epsilon_{\mathrm{L}}=\epsilon_{\perp}^{2}+\epsilon_{\mathrm{x}}^{2}
\end{gathered}
$$

where $\omega_{c}=e B_{z} / m_{e}$ is the cyclotron frequency, $\omega_{p}=\sqrt{n_{e} e^{2} / \epsilon_{0} m_{e}}$ is the plasma frequency, $\omega$ is the angular excitation frequency, $m_{e}$ is the electron mass, $e$ is the electron charge, $B_{z}$ is the magnitude of the axially directed magnetic field, $\epsilon_{0}$ is permittivity of free space, and $n_{e}$ is the electron density.

B. Field Equations for a region of Homogenous Isotropic Dielectric

Maxwell's equations for a region containing a homogenous isotropic dielctric can be written in the form

$$
\begin{gathered}
\nabla_{\perp} E_{z}=i k_{z} \mathbf{E}_{\perp}+i k_{0} Z_{0} \hat{\mathbf{z}} \times \mathbf{H}_{\perp} \\
\nabla_{\perp} \times \mathbf{E}_{\perp}=i k_{0} Z_{0} H_{z} \hat{\mathbf{z}} \\
\nabla_{\perp}\left(Z_{0} H_{z}\right)=i k_{z} Z_{0} \mathbf{H}_{\perp}-i k_{0} \epsilon_{r} \hat{\mathbf{z}} \times \mathbf{E}_{\perp} \\
\nabla_{\perp} \times\left(Z_{0} \mathbf{H}_{\perp}\right)=-i k_{0} \epsilon_{r} E_{z} \hat{\mathbf{z}}
\end{gathered}
$$

where $\mathbf{E}$ and $\mathrm{H}$ are electric and magnetic field intensities respectively, $k_{0}=\omega / c$ is the free space wavenumber, $c$ is the speed of light in vacuum, $k_{z}$ is the component of $k$ parallel to the $z$-axis, $\epsilon_{r}$ is the relative dielectric constant for the region, $Z_{0}=\sqrt{\mu_{0} / \epsilon_{0}}$ is the impedance of free space, and $\mu_{0}$ is the permeability of free space.

The perpendicular components of the fields can be written in terms of the parallel components as follows

$$
\begin{gathered}
\mathbf{E}_{\perp}=-\frac{i}{k_{\perp}^{2}}\left[k_{0} \hat{\mathbf{z}} \times \nabla_{\perp}\left(Z_{0} H_{z}\right)-k_{z} \nabla_{\perp} E_{z}\right] \\
Z_{0} \mathbf{H}_{\perp}=\frac{i}{k_{\perp}^{2}}\left[\epsilon_{r} k_{0} \hat{\mathbf{z}} \times \nabla_{\perp} E_{z}+k_{z} \nabla_{\perp}\left(Z_{0} H_{z}\right)\right]
\end{gathered}
$$

in which $k_{\perp}=\sqrt{k_{0}^{2} \epsilon_{r}-k_{z}^{2}}$ is the perpendicular wavenumber. In simple situations, these equations can be decoupled by assuming that either $E_{z}$ or $H_{z}$ vanish independently to form transverse electric (TE or $\mathrm{H}$ ) modes and transverse magnetic (TM or E) modes respectively. Below, due to the presence of the plasma, the field components are coupled leading to the 
more general $\mathrm{EH}$ and $\mathrm{HE}$ modes. In cylindrical coordinates the general solution may be written in matrix form as

$$
\left(\begin{array}{c}
E_{r} \\
E_{\theta} \\
Z_{h} H_{r} \\
Z_{h} H_{\theta}
\end{array}\right)=i \frac{k_{0} k_{z}}{k_{\perp}^{2}}\left(\begin{array}{cccc}
1 & 0 & 0 & 1 \\
0 & 1 & -1 & 0 \\
0 & -\frac{k_{0}^{2} \epsilon_{r}}{k_{z}^{2}} & 1 & 0 \\
\frac{k_{0}^{2} \epsilon_{r}}{k_{z}^{2}} & 0 & 0 & 1
\end{array}\right)\left(\begin{array}{c}
\frac{1}{k_{0}} \frac{\partial E_{z}}{\partial r} \\
\frac{1}{k_{0} r} \frac{\partial E_{z}}{\partial \theta} \\
\frac{Z_{h}}{k_{0}} \frac{\partial H_{z}}{\partial r} \\
\frac{Z_{h}}{k_{0} r} \frac{\partial H_{z}}{\partial \theta}
\end{array}\right)
$$

where $Z_{h}=Z_{0} k_{0} / k_{z}$. Define

$$
\begin{array}{rc}
\chi_{e m, j}(\rho)=A_{e m}^{(j)} J_{m}(\rho)+B_{e m}^{(j)} Y_{m}(\rho), & \chi_{h m, j}(\rho)=A_{h m}^{(j)} J_{m}(\rho)+B_{h m}^{(j)} Y_{m}(\rho) \\
\chi_{e m, j}^{\prime}(\rho)=\frac{\partial \chi_{e m, j}}{\partial \rho}, & \chi_{h m, j}^{\prime}(\rho)=\frac{\partial \chi_{h m, j}}{\partial \rho}
\end{array}
$$

where $J_{m}$ and $Y_{m}$ are Bessel functions of the first and second kind respectively and $j$ is a region index. The individual fields components for the $m^{\text {th }}$ azimuthal mode are then

$$
\begin{gathered}
E_{r}^{(j)}=\frac{i n_{z}}{\sigma_{j} \nu_{\perp j}^{2}}\left[\nu_{\perp j} \chi_{h m, j}^{\prime}\left(k_{0} \nu_{\perp j} r\right)+\frac{i m}{k_{0} r} \chi_{e m, j}\left(k_{0} \nu_{\perp j} r\right)\right] e^{i m \theta} \\
E_{\theta}^{(j)}=\frac{i n_{z}}{\sigma_{j} \nu_{\perp j}^{2}}\left[\frac{i m}{k_{0} r} \chi_{h m, j}\left(k_{0} \nu_{\perp j} r\right)-\nu_{\perp j} \chi_{e m, j}^{\prime}\left(k_{0} \nu_{\perp j} r\right)\right] e^{i m \theta} \\
Z_{h} H_{r}^{(j)}=\frac{i n_{z}}{\sigma_{j} \nu_{\perp j}^{2}}\left[-\frac{\epsilon_{j}}{n_{z}^{2}} \frac{i m}{k_{0} r} \chi_{h m, j}^{(j)}=\chi_{h m, j}\left(k_{0} \nu_{\perp j} r\right) e^{i m \theta},\right. \\
Z_{h} H_{\theta}^{(j)}=\frac{i n_{z}}{\sigma_{j} \nu_{\perp j}^{2}}\left[\frac{\epsilon_{j}}{n_{z}^{2}} \nu_{\perp j} \chi_{h m, j}^{\prime}\left(k_{\perp j} \nu_{\perp j} r\right)+\frac{i m}{k_{0} r} \chi_{e m, j}^{\prime}\left(k_{0} \nu_{\perp j} r\right)\right] e^{i m \theta} \\
\left.\left.Z_{h} H_{z}^{(j)}=\chi_{e m, j} r\right)\right] e^{i m \theta}
\end{gathered}
$$

where $\nu_{\perp j}=\sqrt{\left|n_{\perp j}^{2}\right|}>0, n_{\perp j}^{2}=\epsilon_{r j}-n_{z}^{2}, \sigma_{j}=\operatorname{sgn}\left(n_{\perp j}^{2}\right), n_{z}^{2}=k_{z}^{2} / k_{0}^{2}, k_{\perp}^{2}=k_{0}^{2} \nu_{\perp j}^{2} \sigma_{j}$ $n_{\perp j}=k_{\perp j} / k_{0}$ and $j$ is a region index. We are interested only in propagating modes and implicitly assume $k_{z}^{2}>0$.

The Bessel functions $J_{m}$ and $Y_{m}$ have been used which are the appropriate choice for $\sigma_{j}>0$. Alternatively, the immaginary counterparts of these functions $\left(I_{m}\right.$ and $\left.K_{m}\right)$ need to be substituted in the event $\sigma_{j}<0$. 


\section{Field Equations for a Magnetized Plasma}

In a region containing a magnetized plasma the field equations are more complicated owing to the tensor dielectric. As above the perpendicular gradients of the field components can be written in terms of the transverse components. The primary differences are in the $H_{z}$ and $\mathrm{H}_{\perp}$ equations.

$$
\begin{aligned}
\nabla_{\perp} E_{z} & =i k_{z} \mathbf{E}_{\perp}+i k_{0} Z_{0} \hat{\mathbf{z}} \times \mathbf{H}_{\perp} \\
\nabla_{\perp} \times \mathrm{E}_{\perp} & =i k_{0} Z_{0} H_{z} \hat{\mathbf{z}} \\
\nabla_{\perp}\left(Z_{0} H_{z}\right) & =i k_{z} Z_{0} \mathrm{H}_{\perp}-i k_{0} \hat{\mathbf{z}} \times\left(\epsilon_{\perp} \cdot \mathbf{E}_{\perp}\right) \\
\nabla_{\perp} \times\left(Z_{0} \mathrm{H}_{\perp}\right) & =-i k_{0} \epsilon_{z} E_{z} \hat{\mathbf{z}}
\end{aligned}
$$

where

$$
\epsilon_{\perp}=\left(\begin{array}{cc}
\epsilon_{\perp} & \epsilon_{\mathbf{x}} \\
-\epsilon_{\mathbf{x}} & \epsilon_{\perp}
\end{array}\right)
$$

In a manner analogous to that outlined by Allis, Buchsbaum, and Bers ${ }^{16}$ coupled differential equations for $E_{z}$ and $H_{z}$ may be obtained. The system of equations takes the form

$$
\begin{gathered}
\left(\nabla_{\perp}^{2}+a\right) E_{z}=b\left(Z_{0} H_{z}\right) \\
\left(\nabla_{\perp}^{2}+c\right) Z_{0} H_{z}=d E_{z}
\end{gathered}
$$

where

$$
\begin{array}{cc}
a=-\left(k_{z}^{2}-k_{0}^{2} \epsilon_{\perp}\right) \frac{\epsilon_{z}}{\epsilon_{\perp}}, & b=k_{z} k_{0} \frac{\epsilon_{\mathbf{x}}}{\epsilon_{\perp}}, \\
c=-\left(k_{z}^{2}-k_{0}^{2} \frac{\epsilon_{R} \epsilon_{L}}{\epsilon_{\perp}}\right), & d=-k_{z} k_{0} \frac{\epsilon_{\mathbf{x}} \epsilon_{z}}{\epsilon_{\perp}} .
\end{array}
$$

Either $E_{z}$ or $H_{z}$ can be eliminated to yield identical fourth order equations which may be written as

$$
\left(\nabla_{\perp}^{2}+p_{1}^{2}\right)\left(\nabla_{\perp}^{2}+p_{2}^{2}\right)\left(\begin{array}{c}
E_{z} \\
H_{z}
\end{array}\right)=0
$$

where $p_{1}^{2}$ and $p_{2}^{2}$ are solutions to the infinite region Appleton-Hartree dispersion relation

$$
p^{4}-(a+c) p^{2}+(a c-b d)=0
$$

We then have

$$
p_{1,2}^{2}=\frac{1}{2}\left[(a+c) \pm \sqrt{(a-c)^{2}+4 b d}\right]
$$


Note that $p_{1}^{2}$ and $p_{2}^{2}$ are functions of $k_{z}$ and effectively constitute a pair of perpendicular wavenumbers for the plasma region.

After some algebra the transverse field components can be written in a form analogous to that of the isotropic solution. Here we have

$$
\left(\begin{array}{c}
E_{\mathbf{r}} \\
E_{\theta} \\
Z_{h} H_{r} \\
Z_{h} H_{\theta}
\end{array}\right)=-i \Delta n_{z}\left(\begin{array}{cccc}
s & \epsilon_{\mathbf{X}} & -\epsilon_{\mathbf{X}} & s \\
-\epsilon_{\mathbf{X}} & s & -s & -\epsilon_{\mathbf{X}} \\
\epsilon_{\mathbf{x}} & t & s & \epsilon_{\mathbf{X}} \\
-t & \epsilon_{\mathbf{X}} & -\epsilon_{\mathbf{x}} & s
\end{array}\right)\left(\begin{array}{c}
\frac{1}{k_{0}} \frac{\partial E_{z}}{\partial r} \\
\frac{1}{k_{r} r} \frac{\partial E_{z}}{\partial \theta} \\
\frac{Z_{h}}{k_{0}} \frac{\partial H_{z}}{\partial r} \\
\frac{Z_{h}}{k_{0} r} \frac{\partial H_{z}}{\partial \theta}
\end{array}\right)
$$

where $s=n_{z}^{2}-\epsilon_{\perp}, t=\frac{\epsilon_{R} \epsilon_{L}}{n_{z}^{2}}-\epsilon_{\perp}$, and $\Delta=1 /\left(s^{2}-\epsilon_{x}^{2}\right)$.

The normal procedure at this point would be to write the general forms for $E_{z}$ and $H_{z}$ and derive the expressions for the transverse field components. However, because of Eqns. (10ab) $E_{z}$ and $H_{z}$ are not independent of one another. In cylindrical coordinates the general solution for $E_{z}$ may be taken as

$$
E_{z}=\left[A_{p m} J_{m}\left(u R_{1} \rho\right)+B_{p m} J_{m}\left(v R_{1} \rho\right)\right] e^{i m \theta}
$$

where $u=p_{1} / k_{0}, v=p_{2} / k_{0}, R_{1}=k_{0} r_{1}, \rho=r / r_{1}$ is the normalized radius, and $r_{1}$ is the plasma radius for our study. The plasma, when present, will always be confined to the innermost region $\left(0 \leq r \leq r_{1}\right)$. Substitution of Eqn. (16a) into Eqn. (10a) then gives for $H_{z}$

$$
Z_{h} H_{z}=\left[\alpha A_{p m} J_{m}\left(u R_{1} \rho\right)+\beta B_{p m} J_{m}\left(v R_{1} \rho\right)\right] e^{i m \theta}
$$

where $\alpha=\left(a-p_{1}^{2}\right) / b n_{z}=d / n_{z}\left(c-p_{2}^{2}\right)$ and $\beta=\left(a-p_{2}^{2}\right) / b n_{z}=d / n_{z}\left(c-p_{1}^{2}\right)$. Define

$$
\begin{gathered}
\chi_{p m, \ell}(\rho)=-\frac{i \Delta n_{z}}{R_{1}}\left[A_{p m} e_{\ell 1} J_{m}\left(u R_{1} \rho\right)+B_{p m} e_{\ell 2} J_{m}\left(v R_{1} \rho\right)\right], \\
\chi_{p m, \ell}^{\prime}(\rho)=-\frac{i \Delta n_{z}}{R_{1}}\left[A_{p m} e_{\ell 1} u J_{m}^{\prime}\left(u R_{1} \rho\right)+B_{p m} e_{\ell 2} v J_{m}^{\prime}\left(v R_{1} \rho\right)\right]
\end{gathered}
$$

where $\ell$ is an index ranging from 1 to 3 and where

$$
\begin{array}{ll}
e_{11}=s-\alpha \epsilon_{\mathrm{x}}, & e_{12}=s-\beta \epsilon_{\mathrm{x}}, \\
e_{21}=\epsilon_{\mathrm{x}}+\alpha s, & e_{22}=\epsilon_{\mathrm{x}}+\beta s, \\
e_{31}=t+\alpha \epsilon_{\mathrm{x}}, & e_{32}=t+\beta \epsilon_{\mathrm{x}} .
\end{array}
$$


The transverse fields may then be compactly written as

$$
\begin{gathered}
E_{r}=\left[R_{1} \chi_{p m, 1}^{\prime}(\rho)+\frac{i m}{\rho} \chi_{p m, 2}(\rho)\right] e^{i m \theta}, \\
E_{\theta}=\left[-R_{1} \chi_{p m, 2}^{\prime}(\rho)+\frac{i m}{\rho} \chi_{p m, 1}(\rho)\right] e^{i m \theta}, \\
Z_{h} H_{r}=\left[R_{1} \chi_{p m, 2}^{\prime}(\rho)+\frac{i m}{\rho} \chi_{p m, 3}(\rho)\right] e^{i m \theta}, \\
Z_{h} H_{\theta}=\left[-R_{1} \chi_{p m, 3}^{\prime}(\rho)+\frac{i m}{\rho} \chi_{p m, 2}(\rho)\right] e^{i m \theta} .
\end{gathered}
$$

\section{Boundary Conditions}

\section{A. Helix Model}

Helix loaded waveguides will be considered below. The sheath helix approximation ${ }^{17}$ is used in the derivation of the dispersion relation involving helices. In this approximation the helix is treated as an anisotropic, infinitely thin cylindrical conductor with infinite conductivity in the direction parallel to a helix of pitch angle, $\psi$, and zero conductivity in the direction perpendicular to the helix angle. The sheath helix boundary conditions are

$$
\mathrm{E}_{\mathrm{v}}^{(1)}=\mathrm{E}_{\mathrm{v}}^{(2)}=0, \quad \mathrm{E}_{\mathrm{w}}^{(1)}=\mathbf{E}_{\mathrm{w}}^{(2)}
$$

where $v$ is the direction parallel to the helix, $w$ is the direction perpendicular to the helix and the superscript refers to field values on either side of the helix. In cylindrical coordinates $(r, \theta, z)$, the helix unit vectors are

$$
\begin{gathered}
\hat{\mathbf{v}}=\hat{\theta} \cos \psi+\hat{\mathbf{z}} \sin \psi \\
\hat{\mathbf{w}}=-\hat{\theta} \sin \psi+\hat{\mathbf{z}} \cos \psi
\end{gathered}
$$

Equations (20ab) are equivalent to the following conditions which are algebraically easier to apply ${ }^{18}$

$$
\mathrm{E}_{\mathrm{v}}^{(1)}=\mathrm{E}_{\mathrm{v}}^{(2)}=0, \quad \mathrm{E}_{\mathrm{z}}^{(1)}=\mathrm{E}_{\mathrm{z}}^{(2)}
$$

The sheath helix model contains the essential physics of the helix without adding undo complexity to the dispersion relation. The primary feature neglected by the sheath helix model is the coupling of azimuthal modes to one another ${ }^{17}$. The azimuthal mode coupling 
results from the boundary condition at a discrete helix and requires the use all normal modes to match the fields at the helix interface. ${ }^{19,20}$

B. Boundary Conditions at a Perfect Conductor

The standard boundary conditions prevail at the interface of a dielectric region and a perfect conductor, namely the vanishing of $\mathbf{E}_{t a n}$ and $\mathbf{H}_{\text {nor }}$ which in cylindrical coordinates become

$$
\begin{gathered}
E_{\theta}\left(a_{c}\right)=0, \quad E_{z}\left(a_{c}\right)=0, \\
H_{r}\left(a_{c}\right)=0
\end{gathered}
$$

where $a_{c}$ is the radius of the conductor.

\section{Boundary Conditions at a Dielectric Interface}

The standard boundary conditions apply at the interface between two regions of differing dielectric constants. These are the continuity of $\mathbf{E}_{t a n}$ and $\mathbf{H}_{t a n}$ across the interface. In cylindrical coordinates these conditions become

$$
\begin{array}{ll}
E_{\theta}^{(1)}\left(a_{d}\right)=E_{\theta}^{(2)}\left(a_{d}\right), & E_{z}^{(1)}\left(a_{d}\right)=E_{z}^{(2)}\left(a_{d}\right) \\
H_{\theta}^{(1)}\left(a_{d}\right)=H_{\theta}^{(2)}\left(a_{d}\right), & H_{z}^{(1)}\left(a_{d}\right)=H_{z}^{(2)}\left(a_{d}\right)
\end{array}
$$

where $a_{d}$ is the radius of the dielectric interface.

\section{Boundary Condition at Infinity}

In order to obtain physical solutions in unbounded regions, the solutions must necessarily vanish at infinity and at a rate such that the integrated power in the mode is finite. The Bessel function satisfying these conditions is $K_{m}$. Thus bounded solutions in an infinite region require both that $\sigma_{\infty}<0$ and that $A_{h m}^{(\infty)}=A_{e m}^{(\infty)}=0$ in Eqns. (7a-f) where the $\infty$ sub- and super-scripts refer to the outermost region.

\section{Derivation of Dispersion Relations}

We now proceed to derive the dispersion relations for a variety of arrangements of generally increasing complexity. Several of the dispersion relations do not contain plasma effects but are included to verify plasma dispersion relations. The derivations also include 
expressions for the field amplitude coefficients, $A_{p m}, B_{p m}, A_{h m}^{(1)}, A_{e m}^{(1)}, B_{e m}^{(1)}, \ldots$, so that field profiles may be obtained for specific solutions to dispersion relations.

\section{A. Single Region Problem (Fig. 1A)}

A particular problem of interest is that of a plasma filled waveguide. The boundary conditions of section III.B will be applied in turn to eliminate the amplitude coefficients $A_{p m}$ and $B_{p m}$. First, the condition on $E_{z}$ yields

$$
B_{p m}=-A_{p m} \frac{J_{m}\left(u R_{1}\right)}{J_{m}\left(v R_{1}\right)}
$$

The condition on $E_{\theta}$, Eqn. (23a) gives

$$
\begin{aligned}
0=-R_{1}\left[A_{p m} e_{21} u J_{m}^{\prime}\left(u R_{1}\right)\right. & \left.+B_{p m} e_{22} v J_{m}^{\prime}\left(v R_{1}\right)\right] \\
& +i m\left[A_{p m} e_{31} J_{m}\left(u R_{1}\right)+B_{p m} e_{32} J_{m}\left(v R_{1}\right)\right]
\end{aligned}
$$

Substitution of Eqn. (25) into (26) yields the dispersion relation, which after some simplification can be written as

$$
\begin{gathered}
0 \equiv D_{0}=e_{21} u R_{1} J_{m}^{\prime}\left(u R_{1}\right) J_{m}\left(v R_{1}\right)-e_{22} v R_{1} J_{m}^{\prime}\left(v R_{1}\right) J_{m}\left(u R_{1}\right) \\
-i m(\beta-\alpha) \epsilon_{\mathrm{x}} J_{m}\left(v R_{1}\right) J_{m}\left(u R_{1}\right)
\end{gathered}
$$

Aside from notational differences, this is the dispersion relation first arrived at by Allis, Buchsbaum, and Bers ${ }^{16}$. Standard circular waveguide modes are recovered in the limit of no plasma $\left(\omega_{p} \rightarrow 0\right)$.

\section{B. Two Region Dispersion Relations}

We consider a two region problem (Fig. 1 ), both with and without a sheath helix separating an outer region, $r_{1}<r<r_{2}$, containing an isotropic dielectric from an inner region, $0<r<r_{1}$, containing either a magnetized plasma or a second isotropic dielectric. To assess the effects of a conducting wall the dispersion relations are also obtained in the limit $r_{2} \rightarrow \infty$.

For the two region case in which the innermost region is plasma filled, the superscript on the outer region amplitude coefficients will be dropped. The regions may be distinguished in these cases by $p, e$, or $h$ appearing in the subscript. 
1. Sheath Helix in a Conducting Cylinder (Fig. 1B)

The conducting cylinder located at $r=r_{2}$ imposes the boundary conditions $E_{z}^{(2)}\left(r_{2}\right)$ $=0$ and $E_{\theta}^{(2)}\left(r_{2}\right)=0$ which respectively yield

$$
B_{h m}^{(2)}=-A_{h m}^{(2)} \frac{I_{m}\left(\nu_{\perp 2} R_{2}\right)}{K_{m}\left(\nu_{\perp 2} R_{2}\right)}
$$

and

$$
B_{e m}^{(2)}=-A_{e m}^{(2)} \frac{I_{m}^{\prime}\left(\nu_{12} R_{2}\right)}{K_{m}^{\prime}\left(\nu_{12} R_{2}\right)} .
$$

The sheath helix condition, $E_{v}^{(1)}\left(r_{1}\right)=E_{v}^{(2)}\left(r_{1}\right)=0$, where $r=r_{1}$ is the radius of the helix gives upon elimination of $B_{e m}^{(2)}$ and $B_{h m}^{(2)}$ respectively

$$
A_{e m}^{(1)}=-A_{h m}^{(1)} \frac{i \sigma_{1} \nu_{\perp 1}}{n_{z}} \Psi_{11} \frac{I_{m}\left(\nu_{\perp 1} R_{1}\right)}{I_{m}^{\prime}\left(\nu_{\perp 1} R_{1}\right)}
$$

and

$$
A_{e m}^{(2)}=-A_{h m}^{(2)} \frac{i \sigma_{2} \nu_{\perp 2}}{n_{z}} \Psi_{21} \frac{K_{m}^{\prime}\left(\nu_{\perp 2} R_{2}\right)}{K_{m}\left(\nu_{\perp 2} R_{2}\right)} \frac{\Upsilon}{\Upsilon^{\prime \prime}}
$$

where

$$
\begin{gathered}
\Psi_{11}=\tan \psi-\frac{m n_{z}}{\sigma_{1} \nu_{\perp 1}^{2} R_{1}}, \quad \Psi_{21}=\tan \psi-\frac{m n_{z}}{\sigma_{2} \nu_{\perp 2}^{2} R_{1}} \\
\Upsilon=I_{m}\left(\nu_{\perp 2} R_{1}\right) K_{m}\left(\nu_{\perp 2} R_{2}\right)-I_{m}\left(\nu_{\perp 2} R_{2}\right) K_{m}\left(\nu_{\perp 2} R_{1}\right) \\
\Upsilon^{\prime \prime}=I_{m}^{\prime}\left(\nu_{\perp 2} R_{1}\right) K_{m}^{\prime}\left(\nu_{\perp 2} R_{2}\right)-I_{m}^{\prime}\left(\nu_{\perp 2} R_{2}\right) K_{m}^{\prime}\left(\nu_{\perp 2} R_{1}\right) .
\end{gathered}
$$

Next the sheath helix condition on the continuity of $E_{z}$ gives upon elimination of $B_{h m}^{(2)}$

$$
A_{h m}^{(2)}=A_{h m}^{(1)} \frac{I_{m}\left(\nu_{\perp 1} R_{1}\right) K_{m}\left(\nu_{\perp 2} R_{2}\right)}{\Upsilon}
$$

The final sheath helix boundary condition on the continuity of $H_{v}$ upon elimination of $A_{e m}^{(1)}, A_{e m}^{(2)}, B_{e m}^{(2)}$ and $B_{h m}^{(2)}$ also yields a relation between $A_{h m}^{(2)}$ and $A_{h m}^{(1)}$, namely

$$
\frac{A_{h m}^{(2)}}{A_{h m}^{(1)}}=\frac{\sigma_{2} \nu_{\perp 2}}{\sigma_{1} \nu_{\perp 1}} \frac{\Upsilon^{\prime \prime} K_{m}\left(\nu_{\perp 2} R_{2}\right)}{I_{m}^{\prime}\left(\nu_{\perp 1} R_{1}\right)} \frac{\epsilon_{1} I_{m}^{\prime 2}\left(\nu_{\perp 1} R_{1}\right)-\nu_{\perp 1}^{2} \Psi_{11}^{2} I_{m}^{2}\left(\nu_{\perp 1} R_{1}\right)}{\epsilon_{2} \Upsilon^{\prime \prime} \Upsilon_{e}-\nu_{\perp 2}^{2} \Psi_{21}^{2} \Upsilon \Upsilon_{h}}
$$

where

$$
\begin{aligned}
& \Upsilon_{e}=I_{m}^{\prime}\left(\nu_{\perp 2} R_{1}\right) K_{m}\left(\nu_{\perp 2} R_{2}\right)-I_{m}\left(\nu_{1.2} R_{2}\right) K_{m}^{\prime}\left(\nu_{\perp 2} R_{1}\right) \\
& \Upsilon_{h}=I_{m}\left(\nu_{\perp 2} R_{1}\right) K_{m}^{\prime}\left(\nu_{\perp 2} R_{2}\right)-I_{m}^{\prime}\left(\nu_{\perp 2} R_{2}\right) K_{m}\left(\nu_{\perp 2} R_{1}\right)
\end{aligned}
$$


Equating expressions (34) and (35) yields the dispersion relation for the sheath helix in a waveguide

$$
\frac{I_{m}\left(\nu_{\perp 1} R_{1}\right) I_{m}^{\prime}\left(\nu_{\perp 1} R_{1}\right)}{\Upsilon \Upsilon^{\prime \prime}}=\frac{\sigma_{2} \nu_{\perp 2}}{\sigma_{1} \nu_{\perp 1}} \frac{\epsilon_{1} I_{m}^{\prime 2}\left(\nu_{\perp 1} R_{1}\right)-\nu_{\perp 1}^{2} \Psi_{11}^{2} I_{m}^{2}\left(\nu_{+1} R_{1}\right)}{\epsilon_{2} \Upsilon^{\prime \prime} \Upsilon_{e}-\nu_{\perp .2}^{2} \Psi_{21}^{2} \Upsilon \Upsilon_{h}}
$$

2. Sheath Helix in an Infinite Medium (Fig. 1C)

The dispersion relation for the sheath helix in an infinite medium can be obtained from Eqn. (36) in the limit $R_{2} \rightarrow \infty$. The result is

$$
\frac{\sigma_{1} \nu_{\perp 1} I_{m}\left(\nu_{\perp 1} R_{1}\right) I_{m}^{\prime}\left(\nu_{11} R_{1}\right)}{\sigma_{2} \nu_{\perp 2} K_{m}\left(\nu_{\perp 2} R_{1}\right) K_{m}^{\prime}\left(\nu_{\perp 2}^{\prime} R_{1}\right)}=\frac{\epsilon_{1} I_{m}^{\prime 2}\left(\nu_{\perp 1} R_{1}\right)-\nu_{\perp 1}^{2} \Psi_{11}^{2} I_{m}^{2}\left(\nu_{\perp 1} R_{1}\right)}{\epsilon_{2} K_{m}^{\prime 2}\left(\nu_{\perp 2} R_{1}\right)-\nu_{\perp 2}^{2} \Psi_{21}^{2} K_{m}^{2}\left(\nu_{\perp 2} R_{1}\right)}
$$

The corresponding field amplitude coefficients in this case are

$$
\begin{gathered}
A_{h m}^{(2)}=A_{e m}^{(2)}=0 \\
A_{e m}^{(1)}=-A_{h m}^{(1)} \frac{i \sigma_{1} \nu_{\perp 1}}{n_{z}} \Psi_{11} \frac{I_{m}\left(\nu_{\perp 1} R_{1}\right)}{I_{m}^{\prime}\left(\nu_{\perp 1} R_{1}\right)} \\
B_{e m}^{(2)}=-B_{h m}^{(2)} \frac{i \sigma_{2} \nu_{\perp 2}}{n_{z}} \Psi_{21} \frac{K_{m}\left(\nu_{\perp 2} R_{1}\right)}{K_{m}^{\prime}\left(\nu_{\perp 2} R_{1}\right)}, \\
B_{e m}^{(2)}=A_{h m}^{(1)} \frac{I_{m}\left(\nu_{\perp 2} R_{1}\right)}{K_{m}\left(\nu_{\perp 1} R_{1}\right)} .
\end{gathered}
$$

In the case that $\epsilon_{1}=\epsilon_{2}=1$, which implies $\nu_{\perp 1}=\nu_{\perp 2}=\nu_{\perp}, \Psi_{11}=\Psi_{21}$, and $\sigma_{1}=\sigma_{2}=$ -1 , we recover, aside from notational changes, the dispersion relation derived by Pierce ${ }^{17,21}$

$$
\frac{I_{m}^{\prime}\left(\nu_{\perp} R_{1}\right) K_{m}^{\prime}\left(\nu_{\perp} R_{1}\right)}{I_{m}\left(\nu_{\perp} R_{1}\right) K_{m}\left(\nu_{\perp} R_{1}\right)}=-\nu_{\perp}^{2}\left(\tan \psi+\frac{m n_{z}}{\nu_{\perp}^{2} R_{1}}\right)^{2}
$$

3. Plasma Column within a Perfectly Conducting Cylinder (Fig. 1D)

For this situation, the plasma is assumed to be confined to the region $0 \leq r \leq r_{1}$ while for $r_{1}<r<r_{2}$ the medium is assumed to be isotropic with dielectric constant $\epsilon_{2}$. Application of boundary conditions Eqn. (23a) and Eqn. (23b) respectively yields

$$
\begin{gathered}
B_{h m}=-A_{h m} \frac{I_{m}\left(\nu_{\perp 2} R_{2}\right)}{K_{m}\left(\nu_{\perp 2} R_{2}\right)}, \\
B_{e m}=-A_{e m} \frac{I_{m}^{\prime}\left(\nu_{\perp 2} R_{2}\right)}{K_{m}^{\prime}\left(\nu_{\perp 2} R_{2}\right)} .
\end{gathered}
$$


From boundary condition Eqn. (24c) and Eqn. (24d) we have

$$
\begin{gathered}
A_{h m}=\frac{K_{m}\left(\nu_{\perp 2} R_{1}\right)}{\Upsilon}\left[A_{p m} J_{m}\left(u R_{1}\right)+B_{p m} J_{m}\left(v R_{1}\right)\right], \\
A_{e m}=\frac{K_{m}^{\prime}\left(\nu_{\perp 2} R_{1}\right)}{\Upsilon_{h}}\left[A_{p m} \alpha J_{m}\left(u R_{1}\right)+B_{p m} \beta J_{m}\left(v R_{1}\right)\right] .
\end{gathered}
$$

From boundary condition Eqn. (24a) we find after eliminating $A_{h m}, B_{h m}, A_{e m}$, and $B_{e m}$

$$
B_{p m}=-A_{p m} \frac{F_{1}(u)+\frac{n_{x}}{\sigma_{2} \nu_{12}}\left[\frac{m}{\nu_{12} R_{1}}+i \alpha \frac{\Upsilon^{\prime \prime}}{\Upsilon_{h}}\right] J_{m}\left(u R_{1}\right)}{G_{1}(v)+\frac{n_{x}}{\sigma_{2} \nu_{\perp 2}}\left[\frac{m}{\nu_{12} R_{1}}+i \beta \frac{\Upsilon^{\prime \prime}}{\Upsilon_{h}}\right] J_{m}\left(v R_{1}\right)}
$$

where

$$
\begin{aligned}
& F_{1}(u)=\frac{i \Delta n_{z}}{R_{1}}\left[e_{21} u R_{1} J_{m}^{\prime}\left(u R_{1}\right)-i m e_{11} J_{m}\left(u R_{1}\right)\right], \\
& G_{1}(v)=\frac{i \Delta n_{z}}{R_{1}}\left[e_{22} v R_{1} J_{m}^{\prime}\left(v R_{1}\right)-i m e_{12} J_{m}\left(v R_{1}\right)\right] .
\end{aligned}
$$

Application of the final boundary condition, Eqn. (24b), and elimination of $A_{h m}, B_{h m}$, $A_{e m}$, and $B_{e m}$ yields another equation containing only $A_{p m}$ and $B_{p m}$, namely

$$
B_{p m}=-A_{p m} \frac{F_{2}(u)+\frac{n_{z}}{\sigma_{2} \nu_{12}}\left[\frac{m \alpha}{\nu_{12} R_{1}}-i \frac{\epsilon_{2}}{n_{2}^{2}} \frac{\Upsilon_{e}}{\Upsilon}\right] J_{m}\left(u R_{1}\right)}{G_{2}(v)+\frac{n_{2}}{\sigma_{2} \nu_{\perp 2}}\left[\frac{m \beta}{\nu_{12} R_{1}}-i \frac{\epsilon_{2}}{n_{z}^{2}} \frac{\Upsilon_{e}}{\Upsilon}\right] J_{m}\left(v R_{1}\right)}
$$

where

$$
\begin{aligned}
& F_{2}(u)=\frac{i \Delta n_{z}}{R_{1}}\left[e_{31} u R_{1} J_{m}^{\prime}\left(u R_{1}\right)-i m e_{21} J_{m}\left(u R_{1}\right)\right], \\
& G_{2}(v)=\frac{i \Delta n_{z}}{R_{1}}\left[e_{32} v R_{1} J_{m}^{\prime}\left(v R_{1}\right)-i m e_{22} J_{m}\left(v R_{1}\right)\right] .
\end{aligned}
$$

Equations (45) and (47) constitute two simultaneous linear equations in two the two unknowns, $A_{p m}$ and $B_{p m}$. The dispersion relation is obtained by their elimination which results in

$$
\begin{aligned}
& \frac{F_{1}(u)+\frac{n_{z}}{\sigma_{2} \nu_{\perp 2}}\left[\frac{m}{\nu_{\perp 2} R_{1}}+i \alpha \frac{\Upsilon^{\prime \prime}}{\Upsilon_{h}}\right] J_{m}\left(u R_{1}\right)}{G_{1}(v)+\frac{n_{z}}{\sigma_{2} \nu_{\perp 2}}\left[\frac{m}{\nu_{\perp 2} R_{1}}+i \beta \frac{\Upsilon^{\prime \prime}}{\Upsilon_{h}}\right] J_{m}\left(v R_{1}\right)}= \\
& \frac{F_{2}(u)+\frac{n_{z}}{\sigma_{2} \nu_{\perp 2}}\left[\frac{m \alpha}{\nu_{12} R_{1}}-i \frac{\epsilon_{2}}{n_{2}^{2}} \frac{\Upsilon_{e}}{\Upsilon}\right] J_{m}\left(u R_{1}\right)}{G_{2}(v)+\frac{n_{2}}{\sigma_{2} \nu_{\perp 2}}\left[\frac{m \beta}{\nu_{\perp 2} R_{1}}-i \frac{\epsilon_{2}}{n_{z}^{2}} \frac{\Upsilon_{e}}{\Upsilon}\right] J_{m}\left(v R_{1}\right)} .
\end{aligned}
$$


4. Plasma Column in an Infinite Medium (Fig. 1E)

To obtain the dispersion relation for the plasma column in an infinite medium take the limit of $R_{2} \rightarrow \infty$. In this case, $\Upsilon_{e} / \Upsilon \sim \Upsilon^{\prime \prime} / \Upsilon_{h} \sim K_{m}^{\prime}\left(\nu_{\perp 2} R_{1}\right) / K_{m}\left(\nu_{\perp_{2}} R_{1}\right)$. The resulting dispersion relation is

$$
\begin{aligned}
& \frac{F_{1}(u)+\frac{n_{z}}{\sigma_{2} \nu_{\perp 2}}\left[\frac{m}{\nu_{\perp 2} R_{1}}+i \alpha \frac{K_{m}^{\prime}\left(\nu_{\perp 2} R_{1}\right)}{K_{m}\left(\nu_{\perp 2} R_{1}\right)}\right] J_{m}\left(u R_{1}\right)}{G_{1}(v)+\frac{n_{z}}{\sigma_{2} \nu_{\perp 2}}\left[\frac{m}{\nu_{\perp 2} R_{1}}+i \beta \frac{K_{m}^{\prime}\left(\nu_{12} R_{1}\right)}{K_{m}\left(\nu_{\perp 2} R_{1}\right)}\right] J_{m}\left(v R_{1}\right)}= \\
& \frac{F_{2}(u)+\frac{n_{z}}{\sigma_{2} \nu_{\perp 2}}\left[\frac{m \alpha}{\nu_{\perp 2} R_{1}}-i \frac{\epsilon_{2}}{n_{z}^{2}} \frac{K_{m}^{\prime}\left(\nu_{\perp 2} R_{1}\right)}{K_{m}\left(\nu_{\perp 2} R_{1}\right)}\right] J_{m}\left(u R_{1}\right)}{G_{2}(v)+\frac{n_{z}}{\sigma_{2} \nu_{\perp 2}}\left[\frac{m \beta}{\nu_{\perp 2} R_{1}}-i \frac{\epsilon_{2}}{n_{z}^{2}} \frac{K_{m}^{\prime}\left(\nu_{\perp 2} R_{1}\right)}{K_{m}\left(\nu_{\perp 2} R_{1}\right)}\right] J_{m}\left(v R_{1}\right)} .
\end{aligned}
$$

The field amplitude coefficients may be found from

$$
\begin{gathered}
A_{h m}=0=A_{e m} \\
\frac{B_{p m}}{A_{p m}}=-\frac{F_{1}(u)+\frac{n_{2}}{\sigma_{2 \nu_{\perp 2}}}\left[\frac{m}{\nu_{\perp 2} R_{1}}+i \alpha \frac{K_{m}^{\prime}\left(\nu_{\perp 2} R_{1}\right)}{K_{m}\left(\nu_{\perp 2} R_{1}\right)}\right]}{G_{1}(v)+\frac{n_{z}}{\sigma_{2} \nu_{\perp 2}}\left[\frac{m}{\nu_{\perp 2} R_{1}}+i \beta \frac{K_{m}^{\prime}\left(\nu_{\perp 2} R_{1}\right)}{K_{m}\left(\nu_{\perp 2} R_{1}\right)}\right]} \\
\frac{B_{h m}}{A_{p m}}=\frac{J_{m}\left(u R_{1}\right)+J_{m}\left(v R_{1}\right) B_{p m} / A_{p m}}{K_{m}\left(\nu_{\perp 2} R_{1}\right)} \\
\frac{B_{e m}}{A_{p m}}=\frac{\alpha J_{m}\left(u R_{1}\right)+\beta J_{m}\left(v R_{1}\right) B_{p m} / A_{p m}}{K_{m}\left(\nu_{\perp 2} R_{1}\right)}
\end{gathered}
$$

5. Plasma Filled Helix in a Conducting Cylinder (Fig. 1F)

As above, the plasma is assumed to be confined to the region $0 \leq r \leq r_{1}$ while for $r_{1}<r<r_{2}$ the medium is assumed to be isotropic with dielectric constant $\epsilon_{2}$. Application of boundary conditions Eqn. (23a) and Eqn. (23b) lead to the same result as above, Eqns. (41) and (42).

Setting $\mathbf{E}_{v}^{(1)}=0$ yields

$$
B_{p m}=-A_{p m} \frac{F_{1}(u)+J_{m}\left(u R_{1}\right) \tan \psi}{G_{1}(v)+J_{m}\left(v R_{1}\right) \tan \psi}
$$

while setting $\mathbf{E}_{\mathbf{v}}^{(2)}=0$ gives upon elimination of $B_{e m}$ and $B_{h m}$

$$
A_{e m}=-i A_{h m} \frac{\sigma_{2} \nu_{\perp 2}}{n_{z}} \frac{\Upsilon}{\Upsilon^{\prime \prime}} \frac{Y_{m}^{\prime}\left(\nu_{\perp 2} R_{2}\right)}{Y_{m}\left(\nu_{\perp 2} R_{2}\right)} \Psi_{21} .
$$


Equating the $z$-component of the electric field on either side of the sheath helix yields upon elimination of $B_{p m}$ and $B_{h m}$

$$
A_{h m}=A_{p m} \frac{Y_{m}\left(\nu_{\perp 2} R_{1}\right)}{\Upsilon} \frac{J_{m}\left(u R_{1}\right) G_{1}(v)-J_{m}\left(v R_{1}\right) F_{1}(u)}{G_{1}(v)+J_{m}\left(v R_{1}\right) \tan \psi} .
$$

This can be simplified by noting that

$$
J_{\mathfrak{m}}\left(u R_{1}\right) G_{1}(v)-J_{m}\left(v R_{1}\right) F_{1}(u)=-\frac{i \Delta n_{z}}{R_{1}} D_{0}
$$

where $D_{0}$ is defined in Eqn. (27) and is the dispersion relation for the plasma filled cylinder. Hence

$$
A_{h m}=-i A_{p m} \frac{\Delta n_{z}}{R_{1}} \frac{Y_{m}\left(\nu_{\perp 2} R_{2}\right)}{\Upsilon} \frac{D_{0}}{G_{1}(v)+J_{m}\left(v R_{1}\right) \tan \psi} .
$$

Finally, the continuity of $\mathrm{H}_{\mathrm{v}}$ across the sheath helix, after elimination of $B_{p m}, B_{e m}$, and $A_{\text {em }}$, results in

$$
A_{h m}=-i A_{p m} \frac{\sigma_{2} \nu_{12} n_{z} \Upsilon^{\prime \prime} Y_{m}\left(\nu_{12} R_{2}\right)}{G_{1}(v)+J_{m}\left(v R_{1}\right) \tan \psi} \frac{\Phi_{1}}{\epsilon_{2} \Upsilon^{\prime \prime} \Upsilon_{e}-\nu_{\perp 2}^{2} \Psi_{21}^{2} \Upsilon \Upsilon_{h}}
$$

where

$$
\begin{aligned}
\Phi_{1}=\left[F_{2}(u)\right. & \left.+\alpha J_{m}\left(u R_{1}\right) \tan \psi\right]\left[G_{1}(v)+J_{m}\left(v R_{1}\right) \tan \psi\right] \\
& -\left[G_{2}(v)+\beta J_{m}\left(v R_{1}\right) \tan \psi\right]\left[F_{1}(u)+J_{m}\left(u R_{1}\right) \tan \psi\right] .
\end{aligned}
$$

Combining the last two results then gives the dispersion relation

$$
\frac{\Delta D_{0}}{R_{1} \Phi_{1}}=\frac{\sigma_{2} \nu_{\perp 2} \Upsilon \Upsilon^{\prime \prime}}{\epsilon_{2} \Upsilon^{\prime \prime} \Upsilon_{e}-\nu_{\perp 2}^{2} \Psi_{21}^{2} \Upsilon \Upsilon_{h}}
$$

Note this form of the dispersion relation isolates plasma terms on left hand side of the equation from the helix terms appearing on the right hand side.

6. Plasma Filled Helix in an Infinite Medium (Fig. 1G)

In this case the field amplitude coefficients are given by

$$
A_{\text {em }}=0=A_{h m} .
$$

The relation between $A_{p m}$ and $B_{p m}$ is unchanged and given by Eqn. (52). We have for the remaining coefficients

$$
B_{e m}=-i B_{h m} \frac{\sigma_{2} \nu_{\perp 2}}{n_{z}} \Psi_{21} \frac{K_{m}\left(\nu_{\perp 2} R_{1}\right)}{K_{m}^{\prime}\left(\nu_{\perp 2} R_{1}\right)}
$$




$$
B_{h m}=-i A_{p m} \frac{\Delta n_{z} D_{0}}{R_{1} K_{m}\left(\nu_{\perp 2} R_{1}\right)} \frac{1}{G_{1}(v)+J_{m}\left(v R_{1}\right) \tan \psi}
$$

The resulting dispersion relation is

$$
\Delta D_{0}\left[\epsilon_{2} K_{m}^{\prime 2}\left(\nu_{\perp 2} R_{1}\right)-\nu_{\perp 2}^{2} \Psi_{21}^{2} K_{m}^{2}\left(\nu_{\perp 2} R_{1}\right)\right]=\sigma_{2} \nu_{\perp 2} R_{1} K_{m}\left(\nu_{\perp 2} R_{1}\right) K_{m}^{\prime}\left(\nu_{\perp 2} R_{1}\right) \Phi_{1}
$$

\section{Three Region Problem (Fig. 1H)}

The three region problem is the most interesting physically, as it represents the most realistic geometry; one in which a vacuum or near vacuum region separates the helix from both the wall and the plasma. This dispersion relation determines how close the plasma must be to the helix in order to obtain good coupling to the plasma wave.

The derivation of the dispersion relation proceeds as above but with much greater complexity due to the added region. Many of the details will be skipped. The dispersion relation which results is a fully populated $4 \times 4$ determinant. Since there is relatively little simplication to be gained in the limit $r_{3} \rightarrow \infty$, this case will not be considered.

The boundary conditions at the wall yield, analogous to Eqns. (41) and (42)

$$
B_{h m}^{(3)}=-A_{h m}^{(3)} \frac{I_{m}\left(\nu_{\perp 3} R_{3}\right)}{K_{m}\left(\nu_{\perp 3} R_{3}\right)}
$$

and

$$
B_{e m}^{(3)}=-A_{e m}^{(3)} \frac{I_{m}^{\prime}\left(\nu_{\perp s} R_{3}\right)}{K_{m}^{\prime}\left(\nu_{\perp 3} R_{3}\right)}
$$

where $R_{3}=k_{0} r_{3}, r_{3}=$ radius of the solid conductor.

The helix boundary conditions for the electric field at $r=r_{2}$, the radius of the helix, gives

$$
\begin{gathered}
A_{e m}^{(3)}=-i A_{h m}^{(3)} \frac{\sigma_{3} \nu_{\perp s}}{n_{z}} \frac{\Upsilon_{23}}{\Upsilon_{23}^{\prime \prime}} \frac{K_{m}^{\prime}\left(\nu_{\perp 3} R_{3}\right)}{K_{m}\left(\nu_{\perp 3} R_{3}\right)} \Psi_{32} \\
-i \frac{\sigma_{2} \nu_{\perp 2} \Upsilon_{23} \Psi_{22}}{n_{z} K_{m}\left(\nu_{\perp 3} R_{3}\right)} A_{h m}^{(3)}=A_{e m}^{(2)} I_{m}^{\prime}\left(\nu_{\perp 2} R_{2}\right)+B_{e m}^{(2)} K_{m}^{\prime}\left(\nu_{\perp 2} R_{2}\right) \\
\frac{\Upsilon_{23}}{K_{m}\left(\nu_{\perp 3} R_{3}\right)} A_{h m}^{(3)}=A_{h m}^{(2)} I_{m}\left(\nu_{\perp 2} R_{2}\right)+B_{h m}^{(2)} K_{m}\left(\nu_{\perp 2} R_{2}\right)
\end{gathered}
$$

where

$$
\begin{gathered}
\Psi_{22}=\tan \psi-\frac{m n_{z}}{\sigma_{2} \nu_{\perp 2}^{2} R_{2}}, \quad \Psi_{32}=\tan \psi-\frac{m n_{z}}{\sigma_{3} \nu_{\perp 3}^{2} R_{2}} \\
\Upsilon_{23}=I_{m}\left(\nu_{\perp 3} R_{2}\right) K_{m}\left(\nu_{\perp 3} R_{3}\right)-I_{m}\left(\nu_{\perp 3} R_{3}\right) K_{m}\left(\nu_{\perp 3} R_{2}\right)
\end{gathered}
$$




$$
\Upsilon_{23}^{\prime \prime}=I_{m}^{\prime}\left(\nu_{\perp s} R_{2}\right) K_{m}^{\prime}\left(\nu_{\perp s} R_{3}\right)-I_{m}^{\prime}\left(\nu_{\perp 3} R_{3}\right) K_{m}^{\prime}\left(\nu_{\perp s} R_{2}\right) .
$$

Next the helix boundary condition for the magnetic field at $r=r_{2}$, the radius of the helix, yields

$$
\begin{aligned}
A_{h m}^{(3)} \frac{i D_{H}}{\sigma_{3} \nu_{\perp 3} n_{z} \Upsilon_{23}^{\prime \prime} K_{m}\left(\nu_{\perp 3} R_{3}\right)}=\frac{i \epsilon_{2}}{\sigma_{2} \nu_{\perp 2} n_{z}} & {\left[A_{h m}^{(2)} I_{m}^{\prime}\left(\nu_{\perp 2} R_{2}\right)+B_{h m}^{(2)} K_{m}^{\prime}\left(\nu_{\perp 2} R_{2}\right)\right] } \\
+ & \Psi_{22}\left[A_{e m}^{(2)} I_{m}\left(\nu_{\perp 2} R_{2}\right)+B_{e m}^{(2)} K_{m}\left(\nu_{\perp 2} R_{2}\right)\right]
\end{aligned}
$$

where the $D_{H}$ is the "helix dispersion relation" given by

$$
D_{H}=\epsilon_{3} \Upsilon_{23 h}^{\prime} \Upsilon_{23}^{\prime \prime}-\nu_{\perp 3}^{2} \Psi_{32}^{2} \Upsilon_{23} \Upsilon_{23 e}^{\prime}
$$

in which

$$
\begin{aligned}
& \Upsilon_{23 h}^{\prime}=I_{m}^{\prime}\left(\nu_{\perp s} R_{2}\right) K_{m}\left(\nu_{\perp s} R_{3}\right)-I_{m}\left(\nu_{\perp s} R_{3}\right) K_{m}^{\prime}\left(\nu_{\perp s} R_{2}\right), \\
& \Upsilon_{23 e}^{\prime}=I_{m}\left(\nu_{\perp s} R_{2}\right) K_{m}^{\prime}\left(\nu_{\perp s} R_{3}\right)-I_{m}^{\prime}\left(\nu_{\perp s} R_{3}\right) K_{m}\left(\nu_{\perp s} R_{2}\right) .
\end{aligned}
$$

Anticipating that linear combinations of the form

$$
A_{h m}^{(2)} I_{m}\left(\nu_{\perp 2} R_{j}\right)+B_{h m}^{(2)} K_{m}\left(\nu_{\perp 2} R_{j}\right)
$$

and its derivative with $j=1,2$ will frequently arise, we digress now to derive some relations which will expedite the remaining algebra. To this end define

$$
\begin{aligned}
& \mathcal{A}_{h j}^{(2)}=A_{h m}^{(2)} I_{m}\left(\nu_{\perp 2} R_{j}\right)+B_{h m}^{(2)} K_{m}\left(\nu_{\perp 2} R_{j}\right), \\
& \mathcal{B}_{h j}^{(2)}=A_{h m}^{(2)} I_{m}^{\prime}\left(\nu_{\perp 2} R_{j}\right)+B_{h m}^{(2)} K_{m}^{\prime}\left(\nu_{\perp 2} R_{j}\right), \\
& \mathcal{A}_{e j}^{(2)}=A_{e m}^{(2)} I_{m}\left(\nu_{\perp 2} R_{j}\right)+B_{e m}^{(2)} K_{m}\left(\nu_{\perp 2} R_{j}\right), \\
& \mathcal{B}_{e j}^{(2)}=A_{e m}^{(2)} I_{m}^{\prime}\left(\nu_{\perp 2} R_{j}\right)+B_{e m}^{(2)} K_{m}^{\prime}\left(\nu_{\perp 2} R_{j}\right) .
\end{aligned}
$$

The $\mathcal{A}$ 's and $\mathcal{B}$ 's subscripted with $j=2$ may be expressed in terms of those subscripted $j=1$ and conversely. The result is

$$
\begin{array}{ll}
\mathcal{A}_{h 1}^{(2)}=-\nu_{\perp 2} R_{2}\left[\Upsilon_{12 e}^{\prime} \mathcal{A}_{h 2}^{(2)}-\Upsilon_{12} \mathcal{B}_{h 2}^{(2)}\right], & \mathcal{B}_{h 1}^{(2)}=-\nu_{\perp 2} R_{2}\left[\Upsilon_{12}^{\prime \prime} \mathcal{A}_{h 2}^{(2)}-\Upsilon_{12 h}^{\prime} \mathcal{B}_{h 2}^{(2)}\right], \\
\mathcal{A}_{e 1}^{(2)}=-\nu_{\perp 2} R_{2}\left[\Upsilon_{12 e}^{\prime} \mathcal{A}_{e 2}^{(2)}-\Upsilon_{12} \mathcal{B}_{e 2}^{(2)}\right], & \mathcal{B}_{e 1}^{(2)}=-\nu_{\perp 2} R_{2}\left[\Upsilon_{12}^{\prime \prime} \mathcal{A}_{e 2}^{(2)}-\Upsilon_{12 h}^{\prime} \mathcal{B}_{e 2}^{(2)}\right], \\
\mathcal{A}_{h 2}^{(2)}=-\nu_{\perp 2} R_{1}\left[\Upsilon_{12 h}^{\prime} \mathcal{A}_{h 1}^{(2)}-\Upsilon_{12} \mathcal{B}_{h 1}^{(2)}\right], & \mathcal{B}_{h 2}^{(2)}=-\nu_{\perp 2} R_{1}\left[\Upsilon_{12}^{\prime \prime} \mathcal{A}_{h 1}^{(2)}-\Upsilon_{12 e}^{\prime} \mathcal{B}_{h 1}^{(2)}\right],
\end{array}
$$




$$
\mathcal{A}_{e 2}^{(2)}=-\nu_{\perp 2} R_{1}\left[\Upsilon_{12 h}^{\prime} \mathcal{A}_{e 1}^{(2)}-\Upsilon_{12} \mathcal{B}_{e 1}^{(2)}\right], \quad \mathcal{B}_{e 2}^{(2)}=-\nu_{\perp 2} R_{1}\left[\Upsilon_{12}^{\prime \prime} \mathcal{A}_{e 1}^{(2)}-\Upsilon_{12 e}^{\prime} \mathcal{B}_{e 1}^{(2)}\right]
$$

where

$$
\begin{aligned}
& \Upsilon_{12}=I_{m}\left(\nu_{\perp 2} R_{1}\right) K_{m}\left(\nu_{\perp 2} R_{2}\right)-I_{m}\left(\nu_{\perp 2} R_{2}\right) K_{m}\left(\nu_{\perp 2} R_{1}\right) \\
& \Upsilon_{12 h}^{\prime}=I_{m}^{\prime}\left(\nu_{\perp 2} R_{1}\right) K_{m}\left(\nu_{\perp 2} R_{2}\right)-I_{m}\left(\nu_{\perp 2} R_{2}\right) K_{m}^{\prime}\left(\nu_{\perp 2} R_{1}\right), \\
& \Upsilon_{12 e}^{\prime}=I_{m}\left(\nu_{\perp 2} R_{1}\right) K_{m}^{\prime}\left(\nu_{\perp 2} R_{2}\right)-I_{m}^{\prime}\left(\nu_{\perp 2} R_{2}\right) K_{m}\left(\nu_{\perp 2} R_{1}\right) . \\
& \Upsilon_{12}^{\prime \prime}=I_{m}^{\prime}\left(\nu_{\perp 2} R_{1}\right) K_{m}^{\prime}\left(\nu_{\perp 2} R_{2}\right)-I_{m}^{\prime}\left(\nu_{\perp 2} R_{2}\right) K_{m}^{\prime}\left(\nu_{\perp 2} R_{1}\right),
\end{aligned}
$$

and where use of the Wronskian

$$
W\left\{I_{m}(z), K_{m}(z)\right\}=I_{m}(z) K_{m}^{\prime}(z)-K_{m}(z) I_{m}^{\prime}(z)=-\frac{1}{z}
$$

and the identity

$$
\Upsilon_{12 e}^{\prime} \Upsilon_{12 h}^{\prime}-\Upsilon_{12} \Upsilon_{12}^{\prime \prime}=-\frac{1}{\nu_{12}^{2} R_{1} R_{2}}
$$

have been made. With the notation introduced above the previous results of Eqns. (66), (67), and (68) can be rewritten as

$$
\begin{gathered}
\mathcal{A}_{h 2}^{(2)}=A_{h m}^{(3)} \frac{\Upsilon_{23}}{K_{m}\left(\nu_{\perp 3} R_{3}\right)} \\
\mathcal{B}_{e 2}^{(2)}=-i \frac{\sigma_{2} \nu_{\perp 2} \Upsilon_{23} \Psi_{22}}{n_{z} K_{m}\left(\nu_{\perp 3} R_{3}\right)} A_{h m}^{(3)}=-i \frac{\sigma_{2} \nu_{\perp 2}}{n_{z}} \Psi_{22} \mathcal{A}_{h 2}^{(2)} \\
\mathcal{B}_{h 2}^{(2)}=\frac{\sigma_{2} \nu_{\perp 2} n_{z}}{i \epsilon_{2}}\left\{\frac{i D_{H}}{\sigma_{3} \nu_{\perp 3} n_{z} \Upsilon_{23} \Upsilon_{23}^{\prime \prime}} \mathcal{A}_{h 2}^{(2)}-\Psi_{22} \mathcal{A}_{\mathrm{e} 2}^{(2)}\right\} .
\end{gathered}
$$

Continuing, the continuity of $E_{\theta}$ at the plasma-vacuum boundary gives

$$
A_{p m} F_{1}(u)+B_{p m} G_{1}(v)+w_{1 h} \mathcal{A}_{h 2}^{(2)}+w_{1 e} \mathcal{A}_{e 2}^{(2)}=0
$$

where

$$
\begin{gathered}
w_{1 h}=-\frac{n_{z} R_{2}}{\sigma_{2} \nu_{\perp 2} R_{1}}\left[m \Upsilon_{12 e}^{\prime}-\frac{m \sigma_{2} \nu_{\perp 2} \Upsilon_{12} D_{H}}{\epsilon_{2} \sigma_{3} \nu_{\perp 3} \Upsilon_{23} \Upsilon_{23}^{\prime \prime}}-\frac{\sigma_{2} \nu_{\perp 2}^{2} R_{1} \Upsilon_{12 h}^{\prime} \Psi_{22}}{n_{z}}\right] \\
w_{1 e}=\frac{i n_{z} R_{2}}{\sigma_{2} \nu_{12} R_{1}}\left[\frac{m \sigma_{2} \nu_{\perp 2} n_{z} \Upsilon_{12} \Psi_{22}}{\epsilon_{2}}-\nu_{\perp 2} R_{1} \Upsilon_{12}^{\prime \prime}\right]
\end{gathered}
$$

Similarly, continuity of $E_{z}$ at the plasma-vacuum interface gives

$$
A_{p m} J_{m}\left(u R_{1}\right)+B_{p m} J_{m}\left(v R_{1}\right)+w_{2 h} \mathcal{A}_{h 2}^{(2)}+w_{2 e} \mathcal{A}_{e 2}^{(2)}=0
$$


in which

$$
\begin{gathered}
w_{2 h}=\nu_{\perp 2} R_{2}\left[\Upsilon_{12 e}^{\prime}-\frac{\sigma_{2} \nu_{\perp 2} \Upsilon_{12} D_{H}}{\epsilon_{2} \sigma_{3} \nu_{\perp 3} \Upsilon_{23} \Upsilon_{23}^{\prime \prime}}\right] \\
w_{2 e}=-i \nu_{\perp 2} R_{2}\left[\frac{\sigma_{2} \nu_{\perp 2} n_{z} \Upsilon_{12} \Psi_{22}}{\epsilon_{2}}\right]
\end{gathered}
$$

The last two boundary conditions, those on the continuity of $H_{\theta}$ and $H_{z}$, respectively yield

$$
\begin{gathered}
A_{p m} F_{2}(u)+B_{p m} G_{2}(v)+w_{3 h} \mathcal{A}_{h 2}^{(2)}+w_{3 e} \mathcal{A}_{e 2}^{(2)}=0 \\
\alpha A_{p m} J_{m}\left(u R_{1}\right)+\beta B_{p m} J_{m}\left(v R_{1}\right)+w_{4 h} \mathcal{A}_{h 2}^{(2)}+w_{4 e} \mathcal{A}_{e 2}^{(2)}=0
\end{gathered}
$$

where

$$
\begin{gathered}
w_{3 h}=\frac{i n_{z} R_{2}}{\sigma_{2}}\left[\frac{\epsilon_{2} \Upsilon_{12}^{\prime \prime}}{n_{z}^{2}}-\frac{\sigma_{2} \nu_{12} \Upsilon_{12 h}^{\prime} D_{H}}{\sigma_{3} \nu_{\perp 3} n_{z}^{2} \Upsilon_{23} \Upsilon_{23}^{\prime \prime}}-\frac{m \sigma_{2} \Upsilon_{12} \Psi_{22}}{n_{z} R_{1}}\right] \\
w_{3 e}=\frac{n_{z} R_{2}}{\sigma_{2} \nu_{12}}\left[\frac{\sigma_{2} \nu_{\perp 2}^{2} \Upsilon_{12 h}^{\prime} \Psi_{22}}{n_{z}}-\frac{m \Upsilon_{12 e}^{\prime}}{R_{1}}\right] \\
w_{4 h}=\nu_{\perp 2} R_{2} \frac{i \sigma_{2} \nu_{12} \Upsilon_{12} \Psi_{22}}{n_{z}} \\
w_{4 e}=\nu_{12} R_{2} \Upsilon_{12 e}^{\prime}
\end{gathered}
$$

Equations (83), (85), (87) and (88) yield the dispersion relation in the form of a $4 \times 4$ determinant

$$
\Delta_{4} \equiv\left|\begin{array}{cccc}
F_{1}(u) & G_{1}(v) & w_{1 h} & w_{1 e} \\
J_{m}\left(u R_{1}\right) & J_{m}\left(v R_{1}\right) & w_{2 h} & w_{2 e} \\
F_{2}(u) & G_{2}(v) & w_{3 h} & w_{3 e} \\
\alpha J_{m}\left(u R_{1}\right) & \beta J_{m}\left(v R_{1}\right) & w_{4 h} & w_{4 e}
\end{array}\right|=0 .
$$

Once a solution to Eqn. (91) has been found the ratio of the amplitude coefficients, $B_{p m} / A_{p m}, \mathcal{A}_{h 2}^{(2)} / A_{p m}$, and $\mathcal{A}_{e 2}^{(2)} / A_{p m}$ may be solved for using any three of the equations forming the determinant, $\Delta_{4}$. From Eqns. (82) and (81) $\mathcal{B}_{h 2}^{(2)} / A_{p m}$ and $\mathcal{B}_{e 2}^{(2)} / A_{p m}$ may be found. From the $\mathcal{A}$ 's and $\mathcal{B}$ 's for $j=2$, the $\mathcal{A}$ 's and $\mathcal{B}$ 's for $j=1$ may be derived from Eqns. (76). Also derivable from either subscripted set of .A's and $\mathcal{B}$ 's are $A_{h m}^{(2)} / A_{p m}$, $B_{h m}^{(2)} / A_{p m}, A_{e m}^{(2)} / A_{p m}$, and $B_{e m}^{(2)} / A_{p m}$ through the use Eqns. (75). Finally, $A_{h m}^{(3)} / A_{p m}$, $B_{h m}^{(3)} / A_{p m}, A_{e m}^{(3)} / A_{p m}$, and $B_{e m}^{(3)} / A_{p m}$ can be found from Eqns. (71), (64), (66) and (65) thus completing the solution of all amplitude coefficients.

\section{References}

1. R.W. Boswell, R. Porteous, A. Prytz, A. Bouchoule, and P. Ranson, Phys. Lett.91A, $163(1982)$. 
2. R.W. Boswell, Plasma Phys. and Controlled Fusion 26, 1147 (1984).

3. F.F. Chen, Plasma Phys. and Controlled Fusion 33, 339 (1991).

4. A. Komori, T. Shoji, K. Miyamoto, J. Kawai, and Y. Kawai, Phys. Fluids B 3, 893 (1991).

5. A.J. Perry, D. Vender, and R.W. Boswell, J. Vac. Sci. and Tech. B 9, 310 (1991).

6. F.F. Chen, J. Vac. Sci. and Tech. A 10, 1389 (1992).

7. P.Y. Zhu and R.W. Boswell, Phys. Rev. Letters 63, 2805 (1989).

8. F.F. Chen, Laser and Particle Beams 7, 551 (1989).

9. J. Musil and F. Zacek, Plasma Phys. 13, 471 (1971).

10. J.H. Booske, W.D. Getty, and R.M. Gilgenbach, Phys. Fluids 28, 3116 (1985).

11. B.H. Quon and R.A. Dandl, Phys. Fluids B 1, 2010 (1989).

12. M. Tanaka, R. Nishimoto, S. Higashi, N. Harada, T. Ohi, A. Komori, and Y. Kawai, J. Phys. Soc. Japan 60, 1600 (1991).

13. D.L. Keil, M.H. Bettenhausen, and N. Hershkowitz, IEEE Trans Plasma Sci. 21, 329 (1993).

14. E.B. Hooper, B.W. Stallard, and M.A. Makowski, Proc. 10th Symp. Space Power and Propulsion, Albuquerque, NM 1993, M. S. El-Genk and M. D. Hoover (eds.), (AIP Conf. Proc. 271, Am. Inst. Phys., N. Y. 1993), pp 1419-24.

15. E.B. Hooper, S.W. Ferguson, M.A. Makowski, B.W. Stallard, and J.L. Power, Proc. 23rd Intern. Electric Propulsion Conf., Seattle, WA 1993 (to be published).

16. W.P. Allis, S.J. Buchsbaum, and A. Bers, Waves in Anisotropic Plasmas, MIT Press, Cambridge, Massachusetts, 1963.

17. J.R. Pierce and P.K. Tien, Proc. I.R.E. 1389 (1954).

18. S.P. Morgan and J.A. Young, Bell System Technical Journal 35, 1348 (1956).

19. H.S. Uhm and J.Y. Choe, J. Appl. Phys. 538483 (1982).

20. H.S. Uhm and J.Y. Choe, IEEE MTT-31, 704 (1983).

21. R.E. Collin, Field Theory of Guilded Waves, McGraw-Hill, New York, 1960. 


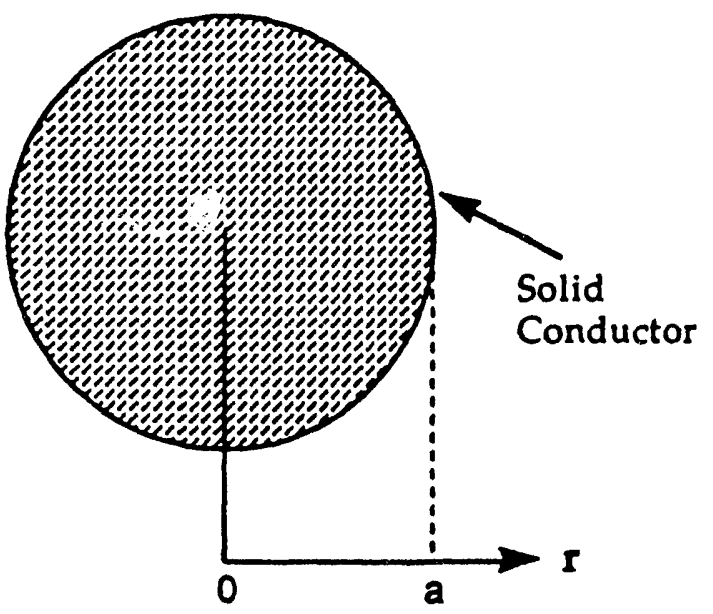

A. Plasma-filled waveguide

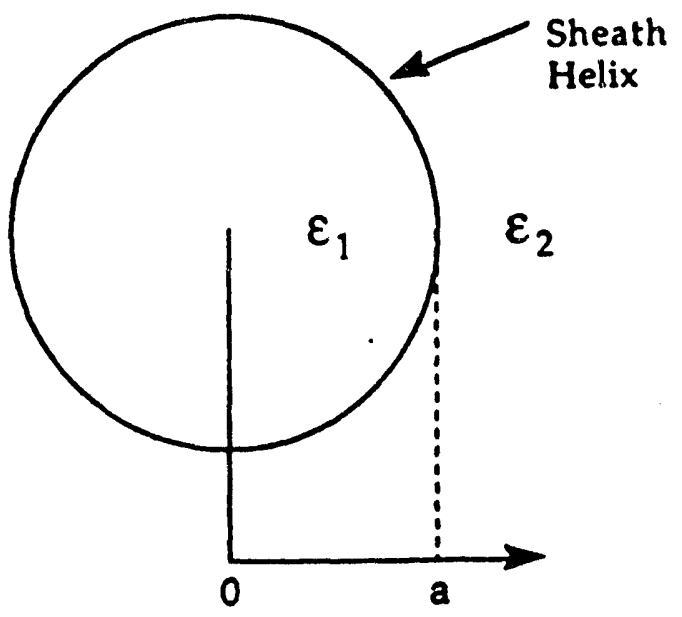

C. Helix in free space

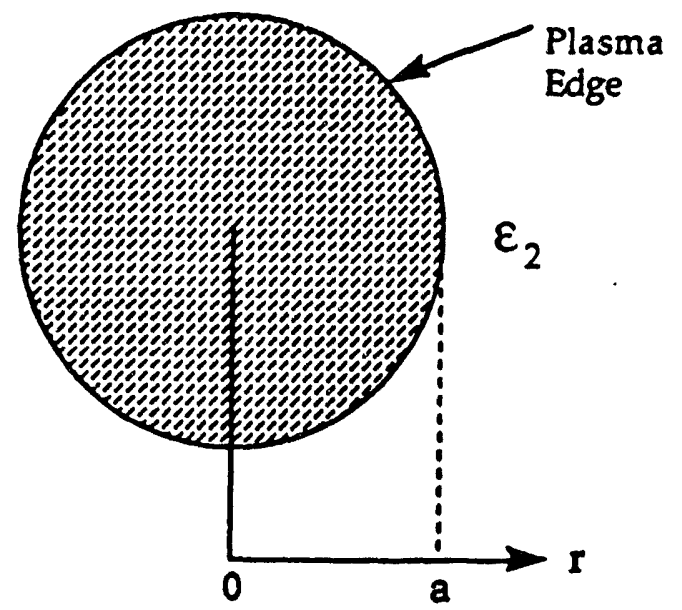

E. Plasma column in free space

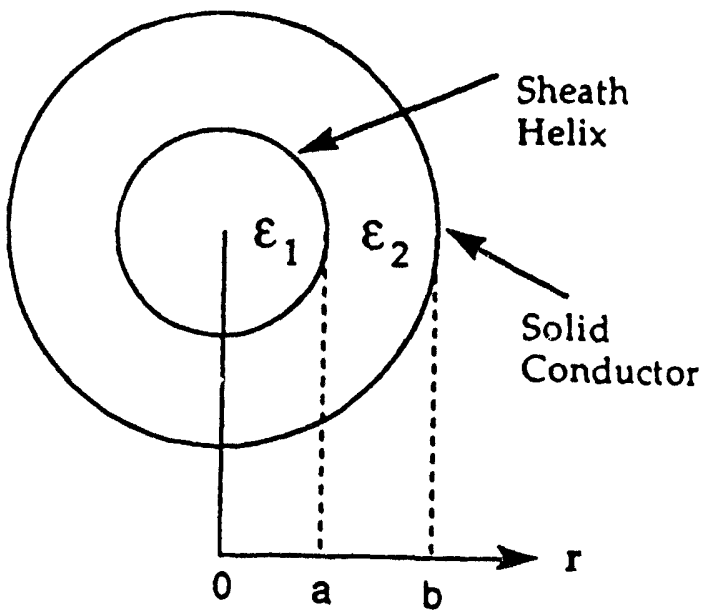

B. Helix-loaded waveguide

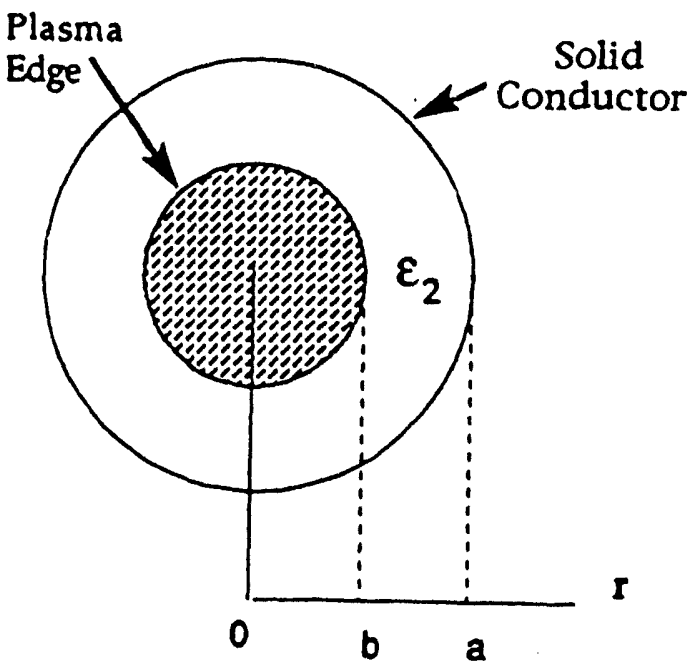

D. Plasma column within a waveguide

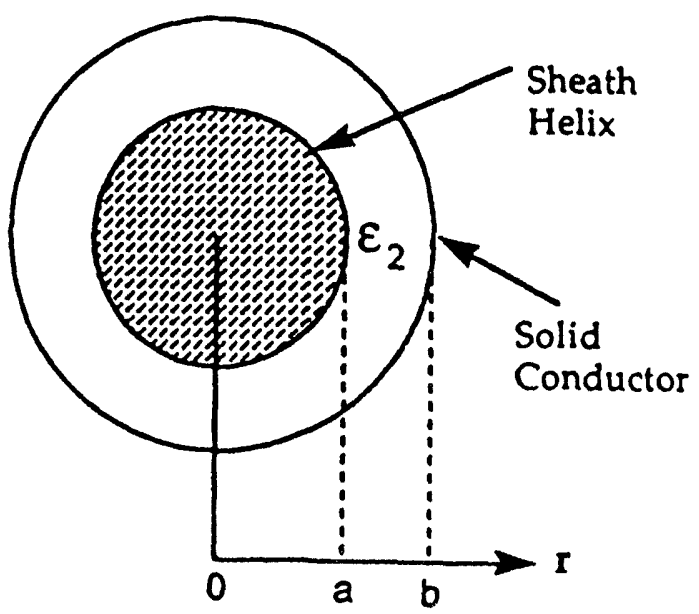

F. Plasma-filled helix in a waveguide 
Solid

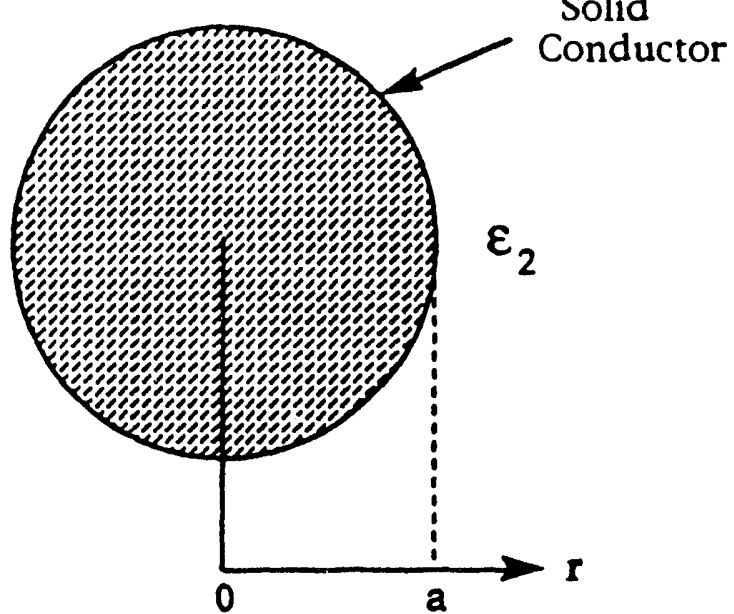

G. Plasma-filled helix in free space

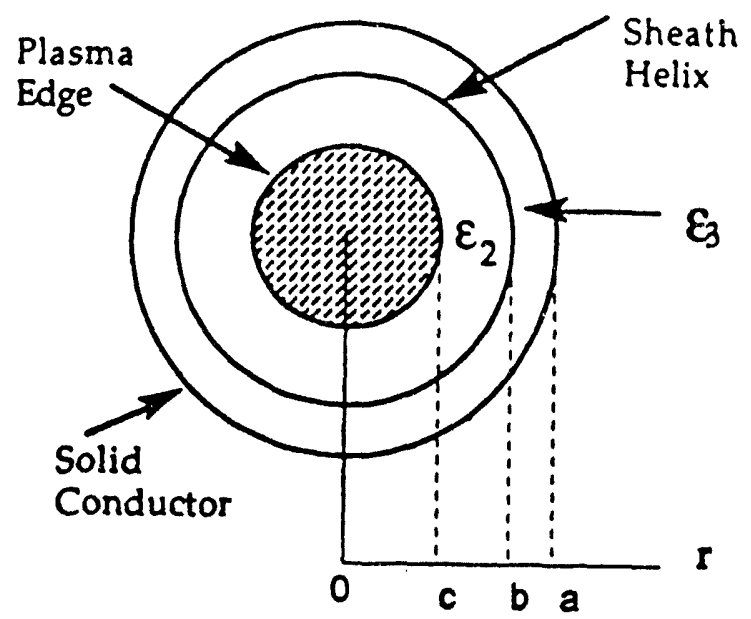

H. Plasma column within a helix within a waveguide

Uniform, Magnetized Plasma

Figure 1. Configuration of the plasma, helix, and waveguide used in the derivation of the dispersion relations. In the figure letters $a, b, c$ replace $r_{i}$ used in the text for region radii. 


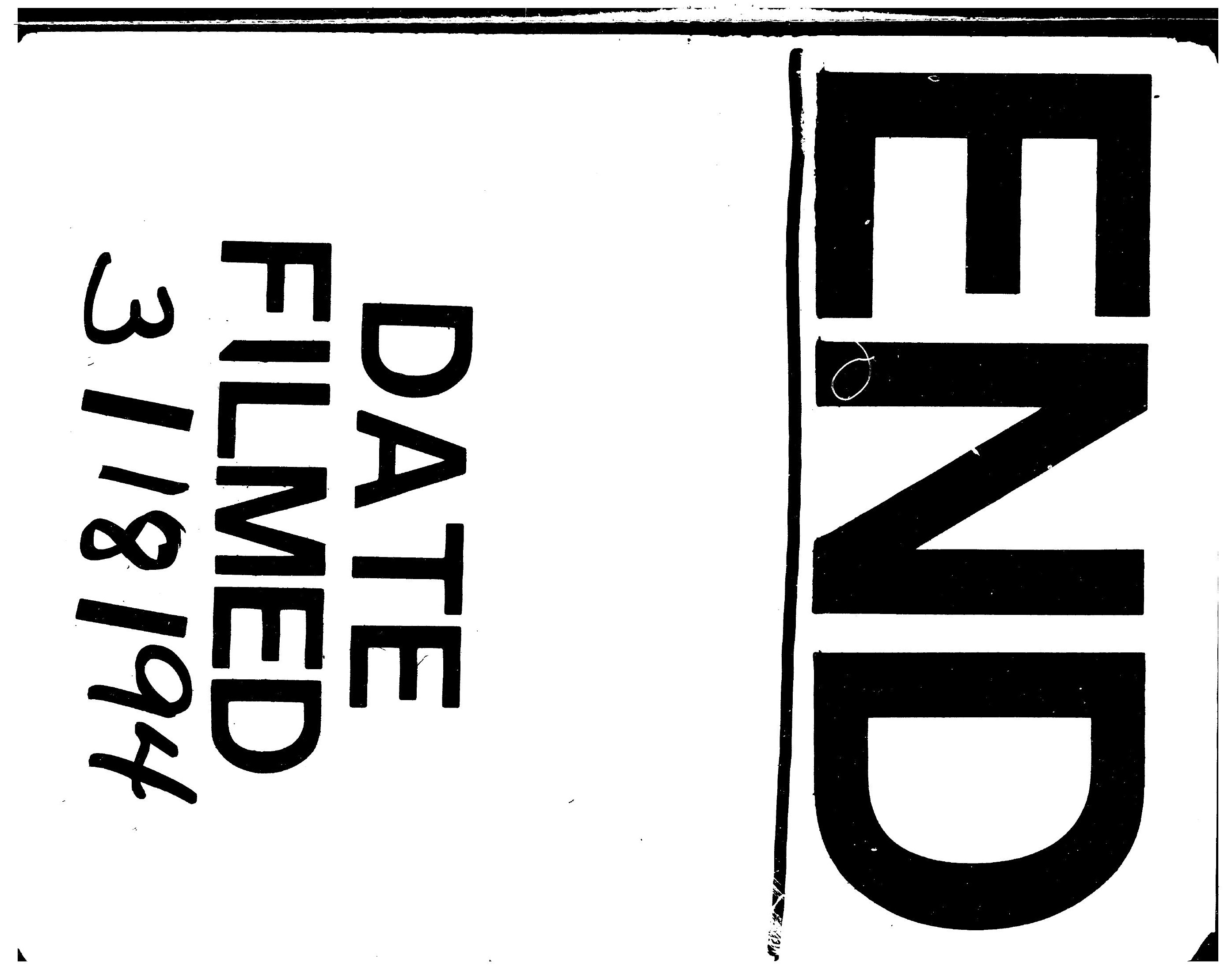


